\title{
Aneurysmal Dilatation of Dialysis Arteriovenous Access
}

\author{
Ravish Shah $^{*}, 1$, Tushar J. Vachharajani ${ }^{2}$ and Anil K. Agarwal ${ }^{1}$ \\ ${ }^{I}$ Division of Nephrology, The Ohio State University, Columbus, Ohio, USA \\ ${ }^{2}$ W. G. (Bill) Hefner Veterans Affairs Medical Center, Salisbury, NC, USA
}

\begin{abstract}
Arteriovenous fistula is considered the "ideal vascular access" in patients with end stage renal disease (ESRD); however, its creation is not without complications. Aneurysmal dilatation is a poorly defined complication which, can potentially lead to access loss and life threatening hemorrhage. Increased venous pressure due to proximal venous stenosis along with repeated cannulations at the same site, are thought to play a significant role in pathogenesis. Given risk of substantial bleeding, it is recommended to avoid cannulation of aneurysmal area; however, as seen in our patient significant numbers of ESRD patients are at risk of being cannulated in the aneurysmal area. Nephrologist taking care of ESRD patients should be aware of its wide clinical spectrum along with signs of "unstable aneurysms" that would mandate an emergent surgical referral. Fistulogram often identifies a high grade proximal stenosis which can be treated by percutaneous angioplasty and perhaps halt the aneurysm progression. Although, endovascular interventions with stent graft placement can be considered in those with pseudoaneurysms related to arteriovenous grafts, it is often an interim measure in an attempt to prolong access longevity. With new surgical techniques and enhanced expertise, it is now possible to salvage the current AV access while simultaneously preserving the precious venous capital for the future AV accesses.
\end{abstract}

Keywords: Dialysis access, arteriovenous access, aneurysm, arteriovenous fistula, arteriovenous graft, pseudoaneurysm.

\section{BACKGROUND}

A well-functioning vascular access (VA) is an absolute requirement to provide life-sustaining dialysis treatment in end stage renal disease (ESRD) patients [1]. Although arteriovenous fistula (AVF) comes the closest to being an ideal vascular access and is often referred to as "lifeline" in these patients, its creation is not without complications [2]. Aneurysmal dilatation is a poorly defined complication of arteriovenous (AV) accesses. The sequelae of aneurysm formation include bleeding, rupture, thrombosis and infection. A thorough physical examination of dialysis arteriovenous access is critical in allowing early diagnosis and timely referral for treatment before complications ensues. Although, NKF-KDOQI guidelines recommend avoiding cannulation of aneurysmal area, a significant number of ESRD patients are being cannulated in the aneurysmal area for variety of reasons, including lack of knowledge of the dialysis staff, lack of awareness amongst practicing nephrologists, poor communication to the dialysis staff and barriers preventing timely referral to name a few. The current article describes a case in which aneurysmal AVF was being cannulated for dialysis due to "lack of cannulation sites" along with a comprehensive review of literature in regards to arteriovenous (AV) access aneurysmal dilatation.

*Address correspondence to this author at the Ohio State University, Division of Nephrology, $395 \mathrm{~W} 12^{\text {th }}$ Avenue, Ground Floor, Columbus, Ohio 43210, USA; Tel: 614293 6839; E-mail: ravish.shah@osumc.edu

\section{CASE REPORT}

A 65-year-old Asian with history of polycystic kidney disease, hypertension, secondary hyperparathyroidism and end stage renal disease (ESRD) was referred to the interventional nephrologist for high venous pressures during dialysis. He had been receiving dialysis through a left forearm AV fistula created 15-years ago. The examination of the AV fistula, revealed two large areas of aneurysmal dilatations close to the arterial anastomosis (Figs. 1, 2). The aneurysmal areas were frequently being cannulated for dialysis given the "lack of cannulation sites". No prior fistulogram was available for comparison. The left forearm fistulogram (with brachial artery cannulation) demonstrated two aneurysmal areas in the outflow cephalic vein close to the AV anastomosis. Additionally, there was $70 \%$ stenosis of the outflow vein, $1-\mathrm{cm}$ proximal to the proximal aneurysmal dilatation, which was successfully treated with percutaneous angioplasty for short term benefits (Fig. 3). In view of the large aneurysmal dilatation and lack of suitable additional segment for cannulation, he was referred for a surgical revision. He underwent resection of the aneurysmal sac, along with luminal reconstruction of the outflow cephalic vein (Fig. 4). However, given the lack of adequate cannulation segment, a short term tunneled central venous catheter was placed during the post surgical recovery period.

\section{DEFINITION}

Aneurysms /pseudoaneurysms are abnormally dilated focal regions of the outflow vein or AVG of varying size and shape [2]. Although there is no uniform definition, an 


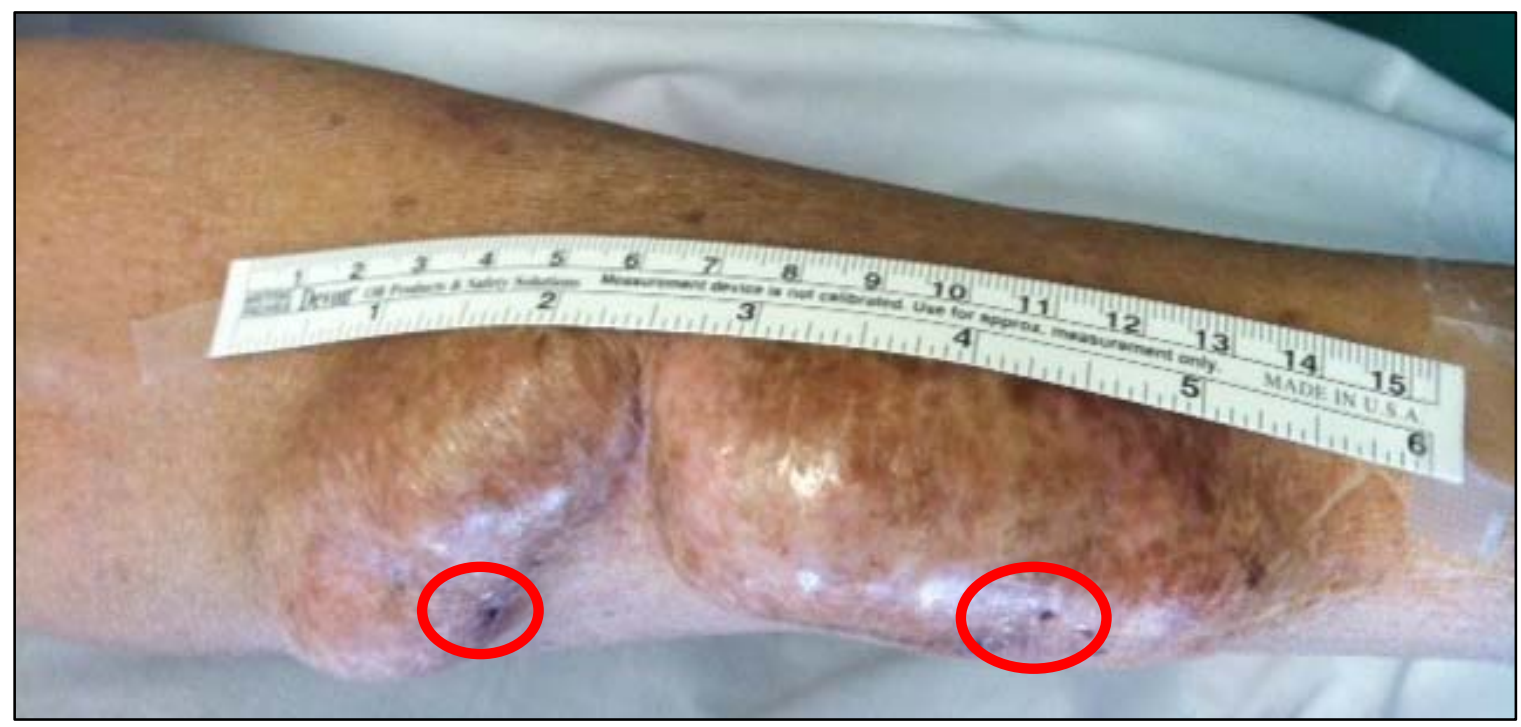

Fig. (1). 65-year-old male with end stage renal disease and a left forearm radio-cephalic arteriovenous fistula with enlarging aneurysms close to the arterial anastomosis, which were being frequently cannulated for dialysis (puncture sites marked by red circles).

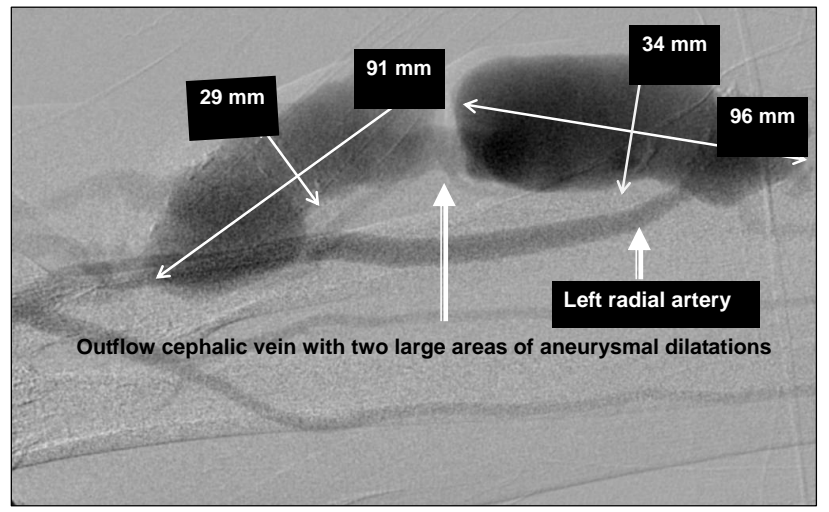

Fig. (2). Left radio-cephalic fistulogram performed with left brachial artery cannulation demonstrating two aneurysmal areas in the outflow cephalic vein close to the arteriovenous anastomosis. (Luminal size noted in millimeters).

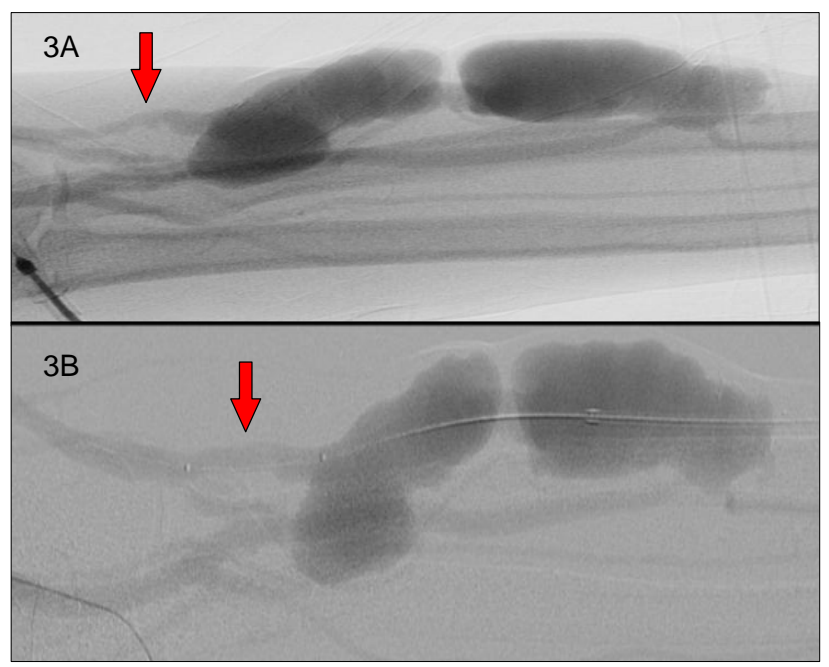

Fig. (3). (A) Outflow cephalic vein stenosis proximal to the aneurysms. (B) Significant improvement in outflow vein stenosis post angioplasty

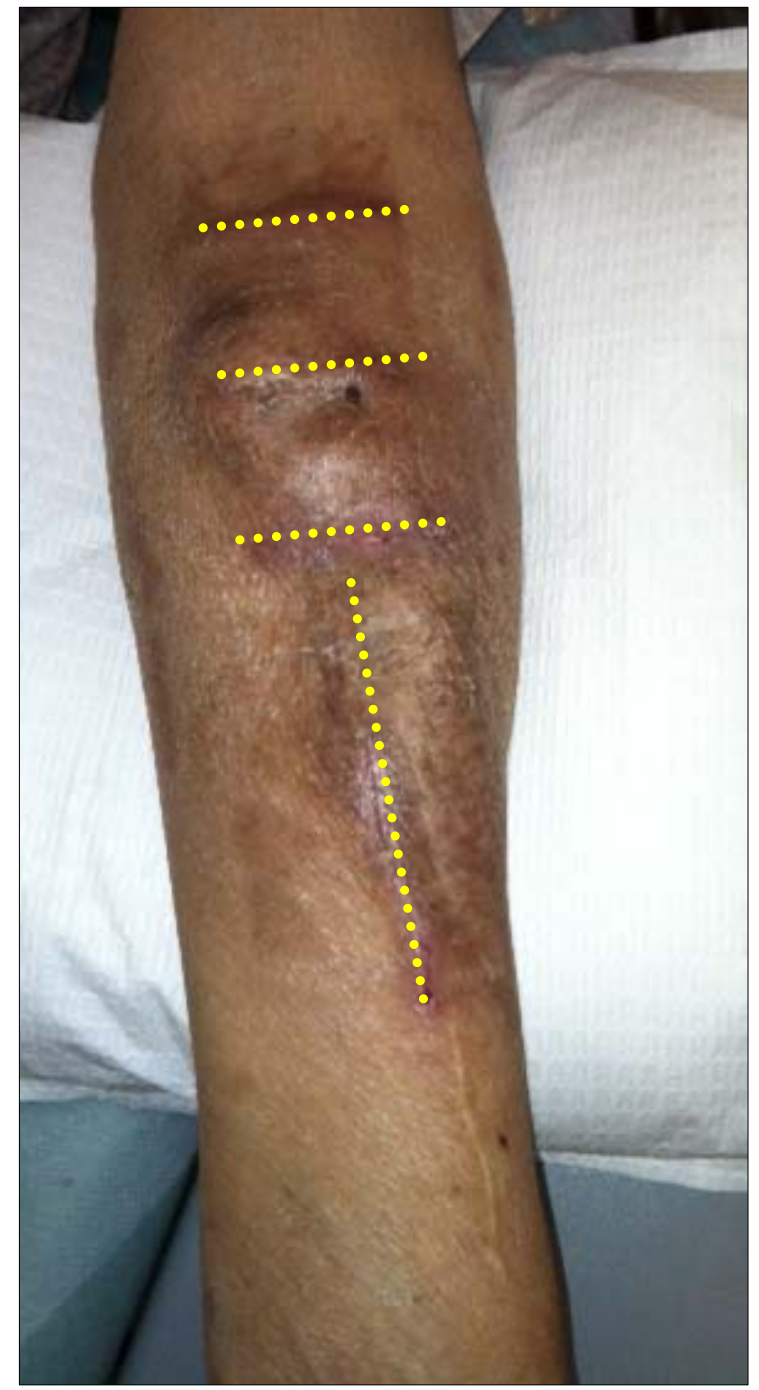

Fig. (4). Four separate incisions were made in the left forearm (marked by dotted lines). The aneurysmal sac was resected and the cephalic vein was over sewn in a longitudinal fashion leaving adequate caliber lumen for cannulation during hemodialysis. 
aneurysm 1.5 to 2 times the size of a native mature vein is considered significant and should be monitored closely [3, 4]. The AVG in the upper extremity has a luminal diameter of $0.6 \mathrm{~cm}$ and the NKF-DOQI guideline recommendation is to refer patients for surgical evaluation if the diameter is 1.5 2.0 times the normal, i.e. $1.2-1.5 \mathrm{~cm} \mathrm{[3].}$

\section{PATHOGENESIS}

Although the real incidence of true aneurysms is unknown; it has been reported to range from 2-30\%, when grouped together with pseudoaneurysms [5]. Long-standing high blood flow rates through an AV fistula results in shear forces damaging the elastic fibers of the internal elastic lamina, often resulting in progressive increase in the size. The high flow rates together with the shear forces from repeated dialysis treatments leads to destruction of the venous wall and aneurysm formation [6,7]. While the precise etiology for aneurysm formation is hard to pin point, increased venous pressure due to presence of down-stream (ante-grade) venous stenosis and repeated cannulations at the same site are thought to be the major contributors $[2,5,8,9]$. The size of this aneurysmal dilatation is directly related to the number of punctures per unit area of cannulation, i.e., the smaller the area, the larger the aneurysmal dilatation [8-10]. A true aneurysm contains all the three layers of vessel wall namely intima, media and adventitia and is frequent with AVF $[2,5]$. In contrast, pseudoaneurysm represent a focal disruption of the vessel wall resulting in collection of blood confined by neointimal or fibrous tissue, generally as a result from repeated cannulation in the same area of the arteriovenous graft (AVG) [2]. Microscopic examination of the true aneurysm demonstrates presence of all three layers of vessel wall along with wall thickening, extensive collagen infiltration with marked derangement of the wall architecture [5].

\section{CLINICAL SPECTRUM}

Although aneurysm formation is a slow process with reported mean time of $3-4$ years for development, it has been reported in patients whose access was never used; suggesting that factors other needle cannulation could play a role [5]. While aneurysmal dilatation is a benign process in most patients, the clinical spectrum can vary widely (Table 1). Surprisingly, questionnaires assessing patient-reported views of vascular access suggest that patients are bothered by the appearance of the fistula more than any other symptom such as pain, bleeding or bruising [11]. AVF rupture may ensue if the aneurysmal segment is directly cannulated, and is precisely the reason for NKF-KDOQI guidelines recommending avoiding venipuncture in the aneurysmal area [4].

\section{ROLE OF PHYSICAL EXAM}

A thorough physical examination is the key to diagnosing AV aneurysms. Aneurysmal dilatations are usually noted as a dilated or ballooned segment of the outflow vein and can be single or multiple. This diffuse, tortuous dilatation should raise the suspicion of a high-grade proximal outflow stenosis [12]. Physical examination of such an obstructed AVF will usually reveal a hyperpulsatile vein, minimal thrill and a high-pitched discontinuous bruit with systolic accentuation and often lacking the diastolic component [12]. In addition, when the extremity is elevated, typically the aneurysmal portion of the fistula distal stenosis remains distended, while the proximal portion collapses in the normal fashion, which can be helpful to localize the site of obstruction [2]. Although there are no standard guidelines, from a practical standpoint, a dilated AV fistula should be evaluated every two weeks, at a minimum. One should look for changes in size along with changes in overlying skin, which can provide clues for an impending rupture. Aneurysms that are unchanged in size overtime and have thick walls are often referred to as "stable aneurysms". One must distinguish stable aneurysms from so called "unstable aneurysm "with characteristic physical findings such as rapidly enlarging, presence of paper thin, shiny, atrophic overlying skin with or without ulcerations and evidence of spontaneous bleeding. An immediate surgical referral is required in patients demonstrating these warning signs indicative of impending catastrophic rupture (Table 2). Currently, there are aneurysm rupture monitoring devices under investigation, which are projected to provide objective measurement of aneurysmal growth and stiffness; the key components predicting aneurysmal rupture. However, it is worth emphasizing that there is no substitute for a periodic thorough vascular access assessment.

\section{Table 1. Clinical Spectrum of AV Access Aneurysms}

* Asymptomatic
* Rapid increase in size
* Difficulty in needle cannulation
* Intra-aneurysmal thrombus formation
* Infection
* Degeneration of the overlying skin
* Heeding or life threatening hemorrhage
* High output cardiac failure

Table 2. Indications for Surgical Referral for AV Access Aneurysms
* Thin, shiny and atrophic overlying skin with or without ulcerations

* Evidence of spontaneous bleeding

* Rapid increase in aneurysmal size

* Evidence of infection

* Limited suitable segment for cannulation

* Cosmetic appearance

\section{MANAGEMENT}

It is crucial to identify patients with "unstable aneurysms" in timely fashion, allowing for an appropriate surgical intervention that would not only prevent aneurysm rupture but also increase the chance of salvaging the existing AV access. In patients with "stable aneurysms", it is critically important to obtain a fistulogram to identify and treat the outflow vein stenosis, since outflow obstruction is often the cause or a contributor to the development and progression of an aneurysm. The treatment options vary 
from conservative monitoring for stable aneurysms / pseudoaneurysms, endovascular interventions such as covered stent graft placement for enlarging pseudoaneurysms and surgical revision for unstable aneurysms.

\section{Conservative Management for Stable Aneurysms}

If an aneurysm has normal overlying skin and does not compromise cannulation, management consists of avoiding cannulating the aneurysm and continued observation. A careful assessment is mandated to identify the cannulation site, away from the aneurysmal area and educating the dialysis staff. A St. Michael's Hospital (SMH) modified buttonhole $(\mathrm{BH})$ cannulation technique was recently proposed as a solution for fistulae with aneurysmal dilatations, in a prospective study of fourteen chronic hemodialysis patients who were followed for 12 months [8]. The protocol included creation of tunnel tracks by experienced cannulators using sharp needles. Once tunneled tracks were established, subsequent cannulation was achieved with dull needles. The cannulation site was carefully selected in areas with maximum skin integrity and carefully avoiding any intra-aneurysmal thrombus by using an onsite ultrasound [8]. The study revealed that by utilizing SMH modified buttonhole technique, post-dialysis needle puncture site hemostasis time decreased from 24 minutes to 15 minutes. In addition, cannulation pain scores decreased significantly. Access flows and dynamic venous pressure measurements remained unchanged and no interventions were required to maintain access patency. The authors concluded that problematic fistulae with large aneurysmal dilatations with a small area available for cannulation can been salvaged by using SMH modified buttonhole technique [8]. However, single center experiences along with nonrandomized design with a small sample size were amongst the significant limitations. In addition, infectious complications were noted in three patients ( 2 cases of septic arthritis and one case of endocarditis- all with Staphylococcus aureus species), raising serious questions related to the safety of this technique and warrants further investigation.

\section{Endovascular Interventions}

Prior to the use of stent grafts, pseudoaneurysm management options were restricted to surgical repair versus access abandonment [13]. Pseudoaneurysm using a stent graft represents an "off-label" use of the device [14]. Use of stent graft in management of pseudoaneurysms poses multiple risks, including recurrence of pseudoaneurysm, stent graft damage as a result of cannulation, and infection. In addition, there are case reports describing the broken stent struts protruding through the skin, potentially posing a threat to the dialysis staff cannulating the patient [14]. In addition to neointimal hyperplasia, repeated cannulation of the stent graft can lead to high intra-graft pressure and recurrence of pseudoaneurysm [13]. Prospective studies comparing safety and efficacy of stent grafts versus surgical approach are long awaited. Based upon the available evidence, stent graft should be considered in patients who are "high surgical risk" or as an interim measure to extend the interval before an eventual surgical intervention [14]. Both patient and dialysis staff must be educated regarding the implications of stent placement, in addition, stent position should be clearly marked and the stent area should be frequently evaluated for evidence of infection or recurrence of pseudoaneurysm [14].

\section{Surgical Intervention}

A multitude of surgical techniques are utilized to repair true aneurysms of native AVF. Although, complete ligation and resection of $\mathrm{AVF}$ is often curative, the access is invariable sacrificed $[15,16]$. In addition, these patients invariably require a tunneled dialysis catheter insertion for continuation of maintenance dialysis while a new AV access is created at another site [15]. Another approach is dissection and ligation of the aneurysmal segment with placement of an interposition greater saphenous vein (GSV) segment or polytetrafluoroethylene (PTFE) graft material [17]. Although, this technique conserves future access sites, it converts an autogenous fistula into a graft [17]. On the other hand surgical resection of excess length, reduction of luminal diameter, and reconstruction has also been shown as a viable option for the treatment of complicated large aneurysms [12, 17]. Although tunneled hemodialysis catheters (THDC) are often required in the post-operative period, use of THDC can sometimes be avoided by cannulating large well matured outflow vein proximal to the aneurysms [10].

\section{CONCLUSIONS}

Aneurysmal dilatation of $\mathrm{AV}$ access represents a unique challenge in ESRD patients and requires a multidisciplinary team work amongst nephrologist, interventionalist, dialysis staff and surgeons, to achieve a timely diagnosis and effective treatment. Although, aneurysms are easily noted on access examination, intervention is often delayed; exposing patients to the significant risks of life threatening hemorrhage. Nephrologist must be aware of the "warning signs" for unstable AV aneurysms and a timely evaluation can not only prevent fatal hemorrhage but increase the chances of salvaging the existing AV access while simultaneously preserving the venous "real estate capital" for future AV access needs.

\section{CONFLICT OF INTEREST}

The authors confirm that this article content has no conflict of interest.

\section{ACKNOWLEDGEMENTS}

Declared none.

\section{REFERENCES}

[1] Shah R, Bhatt UY, Cleef SV, et al. Vascular access thrombosis and interventions in patients missing hemodialysis sessions. Clin Nephrol 2011; 76(6): 435-9.

[2] Beathard GA. Hemodialysis arteriovenous fistulas. 2003. Retrieved from: www.esrdnetwork.org

[3] Vachharajani TJ. Atlas of Dialysis Vascular Access. 2010 Retrieved from: www.fistulafirst.org.

[4] NKF-K/DOQI Clinical Practice Guidelines for Vascular Access. Am J Kidney Dis 2001; 37(1): S147-8.

[5] Pasklinsky G, Meisner RJ, Labropoulos N, et al. Management of true aneurysms of hemodialysis access fistulas. J Vascular Surg 2011; 53(5):1291-7.

[6] Saeed F, Kousar N, Sinnakirouchenan R, et al. Blood loss through AV fistula: a case report and literature review. Int J Nephrol 2011; 2011: 350870 . 
[7] Sultana A, Torella F, McWilliams R, et al. Axillary artery aneurysm following closure of haemodialysis fistula: a case report. J Cardiovasc Surg 2007; 48(2):197-9.

[8] Marticorena RM, Hunter J, Macleod S, et al. The salvage of aneurysmal fistulae utilizing a modified buttonhole cannulation technique and multiple cannulators. Hemodial Int 2006; 10:193200 .

[9] Kro"nung G. Plastic deformation of Cimino fistula by repeated puncture. Dial Transplant 1984; 13: 635-8.

[10] Konner K, Nonnast-Daniel B, Ritz E. The arteriovenous fistula. J Am Soc Nephrol 2003; 14:1669-80.

[11] Quinn RR, Lamping DL, Lok CE, et al. The vascular access questionnaire: assessing patient-reported views of vascular access. J Vasc Access 2008; 9:122-8.
[12] Lawrence M, Roy-Chaudhury P, Asif A, et al. Surgical salvage of the autogenous arteriovenous fistula (AVF). J Nephrol 2007; 20: 388-98.

[13] Niyyar VD, Moossavi S, Vachharajani TJ. Cannulating the hemodialysis access through a stent graft - is it advisable? Clin Nephrol 2012; 77(5): 409-12.

[14] Salman L, Asif A. Stent Graft for Nephrologists: Concerns and Consensus. Clin J Am Soc Nephrol 2010; 5: 1347-52.

[15] Hong-Yee L, Seck-Guan T. Arteriovenous Fistula Aneurysm Plicate, Not Ligate. Ann Acad Med Singapore 2007; 36: 851-3.

[16] Karabay O, Yetkin U, Silistreli E, Uskent H, Onol H, Acikel U. Surgical management of giant aneurysms complicating arteriovenous fistulae. J Int Med Res 2004; 32: 214-7.

[17] Woo K, Cook PR, Joy Garg J, et al. Midterm results of a novel technique to salvage autogenous dialysis access in aneurysmal arteriovenous fistulas. J Vasc Surg 2010; 51: 921-5.

Received: December 5, 2012

Revised: January 10, 2013

Accepted: January 10, 2013

(C) Shah et al.; Licensee Bentham Open.

This is an open access article licensed under the terms of the Creative Commons Attribution Non-Commercial License (http://creativecommons.org/licenses/by-nc/3.0/) which permits unrestricted, non-commercial use, distribution and reproduction in any medium, provided the work is properly cited. 